\title{
Stochastic Stability of Neural Networks with Both Markovian Jump Parameters and Continuously Distributed Delays
}

\author{
Quanxin $\mathbf{Z h u}^{1,2}$ and Jinde Cao ${ }^{1}$ \\ ${ }^{1}$ Department of Mathematics, Southeast University, Nanjing 210096, Jiangsu, China \\ 2 Department of Mathematics, Ningbo University, Ningbo 315211, Zhejiang, China \\ Correspondence should be addressed to Jinde Cao, jdca@seu.edu.cn
}

Received 4 March 2009; Accepted 29 June 2009

Recommended by Manuel De La Sen

The problem of stochastic stability is investigated for a class of neural networks with both Markovian jump parameters and continuously distributed delays. The jumping parameters are modeled as a continuous-time, finite-state Markov chain. By constructing appropriate LyapunovKrasovskii functionals, some novel stability conditions are obtained in terms of linear matrix inequalities (LMIs). The proposed LMI-based criteria are computationally efficient as they can be easily checked by using recently developed algorithms in solving LMIs. A numerical example is provided to show the effectiveness of the theoretical results and demonstrate the LMI criteria existed in the earlier literature fail. The results obtained in this paper improve and generalize those given in the previous literature.

Copyright (C) 2009 Q. Zhu and J. Cao. This is an open access article distributed under the Creative Commons Attribution License, which permits unrestricted use, distribution, and reproduction in any medium, provided the original work is properly cited.

\section{Introduction}

In recent years, neural networks (especially recurrent neural networks, Hopfield neural networks, and cellular neural networks) have been successfully applied in many areas such as signal processing, image processing, pattern recognition, fault diagnosis, associative memory, and combinatorial optimization; see, for example, [1-5]. One of the best important works in these applications is to study the stability of the equilibrium point of neural networks. A major purpose that is concerned with is to find stability conditions (i.e., the conditions for the stability of the equilibrium point of neural networks). To do this, existensive literature has been presented; see, for example, [6-22] and references therein. It should be noted that the methods in the literature have seldom considered the case that the systems have Markovian jump parameters due to the difficulty of mathematics. However, neural networks in real life often have a phenomenon of information latching. 
It is recognized that a way for dealing with this information latching problem is to extract finite state representations (also called modes or clusters). In fact, such a neural network with information latching may have finite modes, and the modes may switch (or jump) from one to another at different times, and the switching (or jumping) between two arbitrarily different modes can be governed by a Markov chain. Hence, the neural networks with Markovian jump parameters are of great significance in modeling a class of neural networks with finite modes.

On the other hand, the time delay is frequently a major source of instability and poor performance in neural networks (e.g., see $[6,23,24]$ ), and so the stability analysis for neural networks with time delays is an important research topic. The existing works on neural networks with time delays can be classified into three categories: constant delays, timevarying delays, and distributed delays. It is noticed that most works in the literature have focused on the former two simple cases: constant delays or time-varying delays (e.g., see $[6,8-10,12-16,19-22])$. However, as pointed out in [18], neural networks usually have a spatial nature due to the presence of a multitude of parallel pathways with a variety of axon sizes and lengths, and so it is desired to model them by introducing continuously distributed delays on a certain duration of time such that the distant past has less influence than the recent behavior of the state. But discussions about the neural networks with continuously distributed delays are only a few researchers $[18,25]$. Therefore, there is enough room to develop novel stability conditions for improvement.

Motivated by the above discussion, the objective of this paper is to study the stability for a class of neural networks with both Markovian jump parameters and continuously distributed delays. Moreover, to make the model more general and practical, the factor of noise disturbance is considered in this paper since noise disturbance is also a major source leading to instability [7]. To the best of the authors' knowledge, up to now, the stability analysis problem for a class of stochastic neural networks with both Markovian jump parameters and continuously distributed delays is still an open problem that has not been properly studied. Therefore, this paper is the first attempt to introduce and investigate the problem of stochastic stability for a class of neural networks with both Markovian jump parameters and continuously distributed delays. By utilizing the Lyapunov stability theory and linear matrix inequality (LMI) technique, some novel delay-dependent conditions are obtained to guarantee the stochastically asymptotic stability of the equilibrium point. The proposed LMI-based criteria are computationally efficient as they can be easily checked by using recently developed standard algorithms such as interior point methods [24] in solving LMIs. Finally, a numerical example is provided to illustrate the effectiveness of the theoretical results and demonstrate the LMI criteria existed in the earlier literature fail. The results obtained in this paper improve and generalize those given in the previous literature.

The remainder of this paper is organized as follows. In Section 2, the model of a class of stochastic neural networks with both Markovian jump parameters and continuously distributed delays is introduced, and some assumptions needed in this paper are presented. By means of Lyapunov-Krasovskii functional approach, our main results are established in Section 3. In Section 4, a numerical example is given to show the effectiveness of the obtained results. Finally, in Section 5, the paper is concluded with some general conclusions.

Notation. Throughout this paper, the following notations will be used. $\mathbb{R}^{n}$ and $\mathbb{R}^{n \times n}$ denote the $n$-dimensional Euclidean space and the set of all $n \times n$ real matrices, respectively. The superscript " $T$ " denotes the transpose of a matrix or vector. Trace $(\cdot)$ denotes the trace of the corresponding matrix, and $I$ denotes the identity matrix with compatible dimensions. For square matrices $M_{1}$ and $M_{2}$, the notation $M_{1}>(\geq,<, \leq) M_{2}$ denotes that $M_{1}-M_{2}$ is 
positive-definite (positive-semidefinite, negative, negative-semidefinite) matrix. Let $w(t)=$ $\left(w_{1}, \ldots, w_{n}\right)^{T}$ be an $n$-dimensional Brownian motion defined on a complete probability space $(\Omega, \mathcal{F}, P)$ with a natural filtration $\left\{\mathcal{F}_{t}\right\}_{t \geq 0}$. Also, let $\tau>0$ and $C\left([-\tau, 0] ; \mathbb{R}^{n}\right)$ denote the family of continuous function $\phi$ from $[-\tau, 0]$ to $\mathbb{R}^{n}$ with the uniform norm $\|\phi\|=$ $\sup _{-\tau \leq \theta \leq 0}|\phi(\theta)|$. Denote by $L_{\mathscr{f}_{t}}^{2}\left([-\tau, 0] ; \mathbb{R}^{n}\right)$ the family of all $\mathcal{F}_{t}$ measurable, $C\left([-\tau, 0] ; \mathbb{R}^{n}\right)$ valued stochastic variables $\xi=\{\xi(\theta):-\tau \leq \theta \leq 0\}$ such that $\int_{-\tau}^{0} E|\xi(s)|^{2} d s<\infty$, where $E[\cdot]$ stands for the correspondent expectation operator with respect to the given probability measure $P$.

\section{Model Description and Problem Formulation}

Let $\{r(t), t \geq 0\}$ be a right-continuous Markov chain on a complete probability space $(\Omega, \mathcal{F}, P)$ taking values in a finite state space $S=\{1,2, \ldots, N\}$ with generator $Q=\left(q_{i j}\right)_{N \times N}$ given by

$$
P\{r(t+\Delta t)=j \mid r(t)=i\}= \begin{cases}q_{i j} \Delta t+o(\Delta t), & \text { if } i \neq j, \\ 1+q_{i i} \Delta t+o(\Delta t), & \text { if } i=j,\end{cases}
$$

where $\Delta t>0$ and $\lim _{\Delta t \rightarrow 0}(o(\Delta t) / \Delta t)=0$. Here, $q_{i j} \geq 0$ is the transition rate from $i$ to $j$ if $i \neq j$ while $q_{i i}=-\sum_{j \neq i} q_{i j}$.

In this paper we consider a class of neural networks with both Markovian jump parameters and continuously distributed delays, which is described by the following integrodifferential equation:

$$
\begin{aligned}
\dot{x}(t)= & -D(r(t)) x(t)+A(r(t)) f(x(t))+B(r(t)) g(x(t-\tau)) \\
& +C(r(t)) \int_{-\infty}^{t} R(t-s) h(x(s)) d s+V
\end{aligned}
$$

where $x(t)=\left[x_{1}(t), x_{2}(t), \ldots, x_{n}(t)\right]^{T}$ is the state vector associated with the $n$ neurons, and the diagonal matrix $D(r(t))=\operatorname{diag}\left(d_{1}(r(t)), d_{2}(r(t)), \ldots, d_{n}(r(t))\right)$ has positive entries $d_{i}(r(t))>0(i=1,2, \ldots, n)$. The matrices $A(r(t))=\left(a_{i j}(r(t))\right)_{n \times n}, B(r(t))=\left(b_{i j}(r(t))\right)_{n \times n}$, and $C(r(t))=\left(c_{i j}(r(t))\right)_{n \times n}$ are, respectively, the connection weight matrix, the discretely delayed connection weight matrix, and the distributively delayed connection weight matrix. $f(x(t))=\left[f_{1}\left(x_{1}(t)\right), f_{2}\left(x_{2}(t)\right), \ldots, f_{n}\left(x_{n}(t)\right)\right]^{T}, g(x(t))=\left[g_{1}\left(x_{1}(t)\right), g_{2}\left(x_{2}(t)\right), \ldots, g_{n}\left(x_{n}(t)\right)\right]^{T}$, and $h(x(t))=\left[h_{1}\left(x_{1}(t)\right), h_{2}\left(x_{2}(t)\right), \ldots, h_{n}\left(x_{n}(t)\right)\right]^{T}$ denote the neuron activation functions, and $V=\left[V_{1}, V_{2}, \ldots, V_{n}\right]^{T}$ denotes a constant external input vector. The constant $\tau>0$ denotes the time delay, and $R=\left[R_{1}, R_{2}, \ldots, R_{n}\right]^{T}$ denotes the delay kernel vector, where $R_{i}$ is a real value nonnegative continuous function defined on $[0, \infty)$ and such that $\int_{0}^{\infty} R_{i}(s) d s=1$ for $i=1,2, \ldots, n$. 
In this paper we will investigate a more general model in which the environmental noise is considered on system (2.2), and so this model can be written as the following integrodifferential equation:

$$
\begin{aligned}
d x(t)=[ & -D(r(t)) x(t)+A(r(t)) f(x(t))+B(r(t)) g(x(t-\tau)) \\
& \left.+C(r(t)) \int_{-\infty}^{t} R(t-s) h(x(s)) d s+V\right] d t+\sigma(x(t), x(t-\tau), t, r(t)) d w(t),
\end{aligned}
$$

where $\sigma: \mathbb{R}^{n} \times \mathbb{R}^{n} \times \mathbb{R}^{+} \times S \rightarrow \mathbb{R}^{n \times n}$ is the noise perturbation.

Throughout this paper, the following conditions are supposed to hold.

Assumption 2.1. There exist six diagonal matrices $U^{-}=\operatorname{diag}\left(u_{1}^{-}, u_{2}^{-}, \ldots, u_{n}^{-}\right), U^{+}=\operatorname{diag}\left(u_{1}^{+}\right.$, $\left.u_{2}^{+}, \ldots, u_{n}^{+}\right), V^{-}=\operatorname{diag}\left(v_{1}^{-}, v_{2}^{-}, \ldots, v_{n}^{-}\right), V^{+}=\operatorname{diag}\left(v_{1}^{+}, v_{2}^{+}, \ldots, v_{n}^{+}\right), W^{-}=\operatorname{diag}\left(w_{1}^{-}, w_{2}^{-}, \ldots, w_{n}^{-}\right)$, and $W^{+}=\operatorname{diag}\left(w_{1}^{+}, w_{2}^{+}, \ldots, w_{n}^{+}\right)$satisfying

$$
\begin{gathered}
u_{i}^{-} \leq \frac{f_{i}(\alpha)-f_{i}(\beta)}{\alpha-\beta} \leq u_{i}^{+}, \\
v_{i}^{-} \leq \frac{g_{i}(\alpha)-g_{i}(\beta)}{\alpha-\beta} \leq v_{i}^{+}, \\
w_{i}^{-} \leq \frac{h_{i}(\alpha)-h_{i}(\beta)}{\alpha-\beta} \leq w_{i}^{+}
\end{gathered}
$$

for all $\alpha, \beta \in \mathbb{R}, \alpha \neq \beta, i=1,2, \ldots, n$.

Assumption 2.2. There exist two positive definite matrices $\Sigma_{1 i}$ and $\Sigma_{2 i}$ such that

$$
\operatorname{trace}\left[\sigma^{T}(x, y, t, r(t)) \sigma(x, y, t, r(t))\right] \leq x^{T} \Sigma_{1 i} x+y^{T} \Sigma_{2 i} y
$$

for all $x, y \in \mathbb{R}^{n}$ and $r(t)=i, i \in S$.

Assumption 2.3. $\sigma(0,0, t, r(t)) \equiv 0$.

Under Assumptions 2.1 and 2.2, it is well known (see, e.g., Mao [16]) that for any initial data $x(\theta)=\xi(\theta)$ on $-\tau \leq \theta \leq 0$ in $L_{\mathscr{f}_{t}}^{2}\left([-\tau, 0] ; \mathbb{R}^{n}\right),(2.3)$ has a unique equilibrium point. Now, let $x^{*}=\left(x_{1}^{*}, x_{2}^{*}, \ldots, x_{n}^{*}\right)$ be the unique equilibrium point of $(2.3)$, and set $y(t)=x(t)-x^{*}$. Then we can rewrite system (2.3) as

$$
\begin{aligned}
d y(t)=[ & -D(r(t)) y(t)+A(r(t)) f^{\prime}(y(t))+B(r(t)) g^{\prime}(y(t-\tau)) \\
& \left.+C(r(t)) \int_{-\infty}^{t} R(t-s) h^{\prime}(y(s)) d s\right] d t+\sigma(y(t), y(t-\tau), t, r(t)) d w(t),
\end{aligned}
$$


where $f^{\prime}(y)=\left[f_{1}^{\prime}\left(y_{1}\right), f_{2}^{\prime}\left(y_{2}\right), \ldots, f_{n}^{\prime}\left(y_{n}\right)\right]^{T}, g^{\prime}(y)=\left[g_{1}^{\prime}\left(y_{1}\right), g_{2}^{\prime}\left(y_{2}\right), \ldots, g_{n}^{\prime}\left(y_{n}\right)\right]^{T}, h^{\prime}(y)=$ $\left[h_{1}^{\prime}\left(y_{1}\right), h_{2}^{\prime}\left(y_{2}\right), \ldots, h_{n}^{\prime}\left(y_{n}\right)\right]^{T}$, and $f_{i}^{\prime}\left(y_{i}\right)=f_{i}\left(y_{i}+x_{i}^{*}\right)-f_{i}\left(x_{i}^{*}\right), g_{i}^{\prime}\left(y_{i}\right)=g_{i}\left(y_{i}+x_{i}^{*}\right)-g_{i}\left(x_{i}^{*}\right)$, $h_{i}^{\prime}\left(y_{i}\right)=h_{i}\left(y_{i}+x_{i}^{*}\right)-h_{i}\left(x_{i}^{*}\right)(i=1,2, \ldots, n)$.

Noting the facts that $f(0)=g(0)=h(0)=0$ and $\sigma(0,0, t, r(t))=0$, the trivial solution of system (2.6) exists. Hence, to prove the stability of $x^{*}$ of (2.3), it is sufficient to prove the stability of the trivial solution of system (2.6). On the other hand, by Assumption 2.1 we have

$$
\begin{gathered}
u_{i}^{-} \leq \frac{f_{i}^{\prime}(\alpha)-f_{i}^{\prime}(\beta)}{\alpha-\beta} \leq u_{i}^{+}, \\
v_{i}^{-} \leq \frac{g_{i}^{\prime}(\alpha)-g_{i}^{\prime}(\beta)}{\alpha-\beta} \leq v_{i}^{+}, \\
w_{i}^{-} \leq \frac{h_{i}^{\prime}(\alpha)-h_{i}^{\prime}(\beta)}{\alpha-\beta} \leq w_{i}^{+}
\end{gathered}
$$

for all $\alpha, \beta \in \mathbb{R}, \alpha \neq \beta, i=1,2, \ldots, n$.

Let $y(t ; \xi)$ denote the state trajectory from the initial data $y(\theta)=\xi(\theta)$ on $-\tau \leq \theta \leq 0$ in $L_{\mathscr{q}_{t}}^{2}\left([-\tau, 0] ; \mathbb{R}^{n}\right)$. Clearly, system $(2.6)$ admits a trivial solution $y(t ; 0) \equiv 0$ corresponding to the initial data $\xi=0$. For simplicity, we write $y(t ; \xi)=y(t)$. Let $C_{1}^{2}\left(\mathbb{R}^{+} \times \mathbb{R}^{n} \times S ; \mathbb{R}^{+}\right)$denote the family of all nonnegative functions $V(t, y, i)$ on $\mathbb{R}^{+} \times \mathbb{R}^{n} \times S$ which are continuously twice differentiable in $y$ and differentiable in $t$. If $V \in C_{1}^{2}\left(\mathbb{R}^{+} \times \mathbb{R}^{n} \times S ; \mathbb{R}^{n}\right)$, then along the trajectory of system (2.6) we define an operator $\mathcal{L} V$ from $\mathbb{R}^{+} \times \mathbb{R}^{n} \times S$ to $\mathbb{R}$ by

$$
\begin{aligned}
\mathcal{L} V(t, y(t), i)=V_{t}(t, y(t), i)+V_{y}(t, y(t), i)\left[\begin{array}{l}
- \\
-
\end{array} \quad+(r(t)) y(t)+A(r(t)) f^{\prime}(y(t))\right. \\
+ \\
\left.+C(r(t)) \int_{-\infty}^{t} R(t-s) h^{\prime}(y(s)) d s\right] \\
+\frac{1}{2} \operatorname{trace}\left[\sigma^{T}(y(t), y(t-\tau), t, r(t)) V_{y y}(t, y(t), i) \sigma(y(t), y(t-\tau), t, r(t))\right] \\
+\sum_{j=1}^{N} q_{i j} V(t, y(t), j),
\end{aligned}
$$

where

$$
\begin{gathered}
V_{t}(t, y(t), i)=\frac{\partial V(t, y(t), i)}{\partial t}, \quad V_{y}(t, y(t), i)=\left(\frac{\partial V(t, y(t), i)}{\partial y_{1}}, \ldots, \frac{\partial V(t, y(t), i)}{\partial y_{n}}\right), \\
V_{y y}(t, y(t), i)=\left(\frac{\partial^{2} V(t, y(t), i)}{\partial y_{i} \partial y_{j}}\right)_{n \times n} .
\end{gathered}
$$

Now we give the definition of stochastic asymptotically stability for system (2.6). 
Definition 2.4. The equilibrium point of (2.6) (or (2.3) equivalently) is said to be stochastic asymptotically stable in the mean square if, for every $\xi \in L_{q_{0}}^{2}\left([-\tau, 0] ; \mathbb{R}^{n}\right)$, the following equality holds:

$$
\lim _{t \rightarrow \infty} E|y(t ; \xi)|^{2}=0
$$

In the sequel, for simplicity, when $r(t)=i$, the matrices $C(r(t)), A(r(t))$, and $B(r(t))$ will be written as $C_{i}, A_{i}$ and $B_{i}$, respectively.

\section{Main Results and Proofs}

In this section, the stochastic asymptotically stability in the mean square of the equilibrium point for system (2.6) is investigated under Assumptions 2.1-2.3.

Theorem 3.1. Under Assumptions 2.1-2.3, the equilibrium point of (2.6) (or (2.3) equivalently) is stochastic asymptotically stable, if there exist positive scalars $\lambda_{i}$, positive definite matrices $G, H, M$, $P_{i}, K_{i}, L_{i}(i \in S)$, and four positive diagonal matrices $Q_{1}, Q_{2}, Q_{3}$, and $F=\operatorname{diag}\left(f_{1}, f_{2}, \ldots, f_{n}\right)$ such that the following LMIs hold:

$$
\left[\begin{array}{cccccccc}
\Gamma_{11} & 0 & P_{i} A_{i} & 0 & 0 & 0 & P_{i} B_{i} & P_{i} C_{i} \\
\star & \Gamma_{22} & 0 & 0 & 0 & 0 & 0 & 0 \\
\star & \star & \Gamma_{33} & 0 & 0 & 0 & 0 & 0 \\
\star & \star & \star & \Gamma_{44} & 0 & 0 & 0 & 0 \\
\star & \star & \star & \star & -Q_{3}+F & 0 & 0 & 0 \\
\star & \star & \star & \star & \star & -K_{i} & 0 & 0 \\
\star & \star & \star & \star & \star & \star & -L_{i} & 0 \\
\star & \star & \star & \star & \star & \star & \star & -F
\end{array}\right]<0,
$$

where the symbol " $\star$ " denotes the symmetric term of the matrix:

$$
\begin{aligned}
\Gamma_{11} & =-2 P_{i} D_{i}+G+\lambda_{i} \Sigma_{1 i}+U Q_{1} U+V Q_{2} V+W Q_{3} W+\sum_{j=1}^{N} q_{i j} P_{j}, \\
\Gamma_{22} & =-G+\lambda_{i} \Sigma_{2 i}, \quad \Gamma_{33}=-Q_{1}+K_{i}+\tau H, \quad \Gamma_{44}=-Q_{2}+L_{i}+\tau M, \\
U & =\operatorname{diag}\left(u_{1}, u_{2}, \ldots, u_{n}\right), \quad V=\operatorname{diag}\left(v_{1}, v_{2}, \ldots, v_{n}\right), \quad W=\operatorname{diag}\left(w_{1}, w_{2}, \ldots, w_{n}\right), \\
u_{i} & =\max \left\{\left|u_{i}^{-}\right|,\left|u_{i}^{+}\right|\right\}, \quad v_{i}=\max \left\{\left|v_{i}^{-}\right|,\left|v_{i}^{+}\right|\right\}, \quad w_{i}=\max \left\{\left|w_{i}^{-}\right|,\left|w_{i}^{+}\right|\right\} \quad(i=1,2, \ldots, n) .
\end{aligned}
$$


Proof. Fixing $\xi \in L_{\mathscr{q}_{t}}^{2}\left([-\tau, 0] ; \mathbb{R}^{n}\right)$ arbitrarily and writing $y(t ; \xi)=y(t)$, consider the following Lyapulov-Krasovskii functional:

$$
V(t, y(t), i)=\sum_{k=1}^{7} V_{k}(t, y(t), i)
$$

where

$$
\begin{aligned}
& V_{1}(t, y(t), i)=y^{T}(t) P_{i} y(t), \\
& V_{2}(t, y(t), i)=\int_{t-\tau}^{t} f^{\prime T}(y(s)) K_{i} f^{\prime}(y(s)) d s, \\
& V_{3}(t, y(t), i)=\int_{-\tau}^{0} d \theta \int_{t+\theta}^{t} f^{\prime T}(y(s)) H f^{\prime}(y(s)) d s, \\
& V_{4}(t, y(t), i)=\sum_{j=1}^{n} f_{j} \int_{0}^{\infty} R_{j}(\theta) \int_{t-\theta}^{t} h_{j}^{\prime 2}\left(y_{j}(s)\right) d s d \theta, \\
& V_{5}(t, y(t), i)=\int_{t-\tau}^{t} y^{T}(s) G y(s) d s, \\
& V_{6}(t, y(t), i)=\int_{t-\tau}^{t} g^{\prime T}(y(s)) L_{i} g^{\prime}(y(s)) d s, \\
& V_{7}(t, y(t), i)=\int_{-\tau}^{0} d \theta \int_{t+\theta}^{t} g^{\prime T}(y(s)) M g^{\prime}(y(s)) d s .
\end{aligned}
$$

For simplicity, denote $\sigma(y(t), y(t-\tau), t, r(t))$ by $\sigma(t)$. Then it follows from (2.10) and (2.6) that

$$
\begin{aligned}
\mathcal{L} V_{1}(t, y(t), i)= & 2 y^{T}(t) P_{i}\left[-D_{i} y(t)+A_{i} f^{\prime}(y(t))+B_{i} g^{\prime}(y(t-\tau))\right. \\
& \left.\quad+C_{i} \int_{-\infty}^{t} R(t-s) h^{\prime}(y(s)) d s\right]+\sum_{j=1}^{N} q_{i j} y^{T}(t) P_{j} y(t)+\operatorname{trace}\left[\sigma^{T}(t) P_{i} \sigma(t)\right] \\
= & y^{T}(t)\left(-2 P_{i} D_{i}\right) y(t)+2 y^{T}(t) P_{i} A_{i} f^{\prime}(y(t))+2 y^{T}(t) P_{i} B_{i} g^{\prime}(y(t-\tau)) \\
& +2 y^{T}(t) P_{i} C_{i} \int_{-\infty}^{t} R(t-s) h^{\prime}(y(s)) d s+y^{T}(t) \sum_{j=1}^{N} q_{i j} P_{j} y(t) \\
& +\operatorname{trace}\left[\sigma^{T}(t) P_{i} \sigma(t)\right]
\end{aligned}
$$

On the other hand, by Assumption 2.2 and condition (3.2) we obtain

$$
\operatorname{trace}\left[\sigma^{T}(t) P_{i} \sigma(t)\right] \leq \lambda_{i} \operatorname{trace}\left[\sigma^{T}(t) \sigma(t)\right] \leq \lambda_{i} y^{T}(t) \Sigma_{1 i} y(t)+\lambda_{i} y^{T}(t-\tau) \Sigma_{2 i} y(t-\tau),
$$


which together with (3.8) gives

$$
\begin{aligned}
\mathcal{L} V_{1}(t, y(t), i) \leq & y^{T}(t)\left(-2 P_{i} D_{i}\right) y(t)+2 y^{T}(t) P_{i} A_{i} f^{\prime}(y(t))+2 y^{T}(t) P_{i} B_{i} g^{\prime}(y(t-\tau)) \\
& +2 y^{T}(t) P_{i} C_{i} \int_{-\infty}^{t} R(t-s) h^{\prime}(y(s)) d s+y^{T}(t) \sum_{j=1}^{N} q_{i j} P_{j} y(t) \\
& +\lambda_{i} y^{T}(t) \Sigma_{1 i} y(t)+\lambda_{i} y^{T}(t-\tau) \Sigma_{2 i} y(t-\tau) .
\end{aligned}
$$

Also, from direct computations, it follows that

$$
\begin{aligned}
\mathcal{L} V_{2}(t, y(t), i)= & f^{\prime T}(y(t)) K_{i} f^{\prime}(y(t))-f^{\prime T}(y(t-\tau)) K_{i} f^{\prime}(y(t-\tau)) \\
& +\int_{t-\tau}^{t} f^{\prime T}(y(s))\left(\sum_{j=1}^{N} q_{i j} K_{j}\right) f^{\prime}(y(s)) d s, \\
\mathcal{L} V_{3}(t, y(t), i)= & \tau f^{\prime T}(y(t)) H f^{\prime}(y(t))-\int_{t-\tau}^{t} f^{\prime T}(y(s)) H f^{\prime}(y(s)) d s, \\
\mathcal{L} V_{4}(t, y(t), i)= & \sum_{j=1}^{n} f_{j} \int_{0}^{\infty} R_{j}(\theta) h_{j}^{\prime 2}\left(y_{j}(t)\right) d \theta-\sum_{j=1}^{n} f_{j} \int_{0}^{\infty} R_{j}(\theta) h_{j}^{\prime 2}\left(y_{j}(t-\theta)\right) d \theta \\
= & h^{\prime T}(y(t)) F h^{\prime}(y(t))-\sum_{j=1}^{n} f_{j} \int_{0}^{\infty} R_{j}(\theta) d \theta \int_{0}^{\infty} R_{j}(\theta) h_{j}^{\prime 2}\left(y_{j}(t-\theta)\right) d \theta \\
\leq & h^{\prime T}(y(t)) F h^{\prime}(y(t))-\sum_{j=1}^{n} f_{j}\left[\int_{0}^{\infty} R_{j}(\theta) h_{j}^{\prime}\left(y_{j}(t-\theta)\right) d \theta\right]^{2} \\
= & h^{\prime T}(y(t)) F h^{\prime}(y(t)) \\
& -\left(\int_{-\infty}^{t} R(t-s) h^{\prime}(y(s)) d s\right)^{T} F \int_{-\infty}^{t} R(t-s) h^{\prime}(y(s)) d s, \\
& +\int_{t-\tau}^{t} g^{\prime T}(y(s))\left(\sum_{j=1}^{N} q_{i j} L_{j}\right)_{g^{\prime}}(y(s)) d s, \\
\mathcal{L} V_{6}(t, y(t), i)= & g^{\prime T}(y(t)) L_{i} g^{\prime}(y(t))-g^{\prime T}(y(t-\tau)) L_{i} g^{\prime}(y(t-\tau)) \\
\mathcal{L} V_{5}(t, y(t), i)= & y^{T}(t) G y(t)-y^{T}(t-\tau) G y(t-\tau), \\
&
\end{aligned}
$$


It should be mentioned that the calculation of $\mathcal{L} V_{4}(t, y(t), i)$ has applied the following inequality:

$$
\left[\int_{0}^{\infty} l_{1}(\theta) l_{2}(\theta) d \theta\right]^{2} \leq \int_{0}^{\infty} l_{1}^{2}(\theta) d \theta \int_{0}^{\infty} l_{2}^{2}(\theta) d \theta
$$

with $l_{1}(\theta):=[R(\theta)]^{1 / 2}$ and $l_{2}(\theta):=[R(\theta)]^{1 / 2} h^{\prime}(y(t-\theta))$.

Furthermore, it follows from the conditions (3.3) and (3.4) that

$$
\begin{aligned}
& \int_{t-\tau}^{t} f^{T}(y(s))\left(\sum_{j=1}^{N} q_{i j} K_{j}\right) f^{\prime}(y(s)) d s-\int_{t-\tau}^{t} f^{T}(y(s)) H f^{\prime}(y(s)) d s \leq 0, \\
& \int_{t-\tau}^{t} g^{\prime T}(y(s))\left(\sum_{j=1}^{N} q_{i j} L_{j}\right) g^{\prime}(y(s)) d s-\int_{t-\tau}^{t} g^{\prime T}(y(s)) M g^{\prime}(y(s)) d s \leq 0 .
\end{aligned}
$$

On the other hand, by Assumption 2.1 we have

$$
\begin{aligned}
& f^{\prime T}(y(t)) Q_{1} f^{\prime}(y(t)) \leq y^{T}(t) U Q_{1} U y(t), \\
& g^{\prime T}(y(t)) Q_{2} g^{\prime}(y(t)) \leq y^{T}(t) V Q_{2} V y(t) \\
& h^{\prime T}(y(t)) Q_{3} h^{\prime}(y(t)) \leq y^{T}(t) W Q_{3} W y(t) .
\end{aligned}
$$

Hence, by (3.8)-(3.14), we get

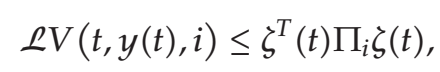

where

$$
\begin{aligned}
& \zeta^{T}(t)=\left[\begin{array}{lllll}
y^{T}(t) & y^{T}(t-\tau) & f^{T}(y(t)) & g^{\prime T}(y(t)) & h^{\prime T}(y(t))
\end{array}\right. \\
& \left.f^{\prime T}(y(t-\tau)) \quad g^{\prime T}(y(t-\tau)) \quad\left(\int_{-\infty}^{t} R(t-s) h^{\prime}(y(s)) d s\right)^{T}\right]
\end{aligned}
$$




$$
\begin{gathered}
\Pi_{i}=\left[\begin{array}{cccccccc}
\Gamma_{11} & 0 & P_{i} A_{i} & 0 & 0 & 0 & P_{i} B_{i} & P_{i} C_{i} \\
\star & \Gamma_{22} & 0 & 0 & 0 & 0 & 0 & 0 \\
\star & \star & \Gamma_{33} & 0 & 0 & 0 & 0 & 0 \\
\star & \star & \star & \Gamma_{44} & 0 & 0 & 0 & 0 \\
\star & \star & \star & \star & -Q_{3}+F & 0 & 0 & 0 \\
\star & \star & \star & \star & \star & -K_{i} & 0 & 0 \\
\star & \star & \star & \star & \star & \star & -L_{i} & 0 \\
\star & \star & \star & \star & \star & \star & \star & -F
\end{array}\right], \\
\Gamma_{11}=-2 P_{i} D_{i}+G+\lambda_{i} \Sigma_{1 i}+U Q_{1} U+V Q_{2} V+W Q_{3} W+\sum_{j=1}^{N} q_{i j} P_{j}, \\
\Gamma_{22}=-G+\lambda_{i} \Sigma_{2 i}, \Gamma_{33}=-Q_{1}+K_{i}+\tau H, \quad \Gamma_{44}=-Q_{2}+L_{i}+\tau M .
\end{gathered}
$$

By condition (3.1), there must exist a scalar $\beta_{i}>0(i \in S)$ such that $\Pi_{i}+\beta_{i} I<0$. Setting $\beta=\min _{i \in S} \beta_{i}$, it is clear that $\beta>0$. Taking the mathematical expectation on both sides of (3.15), we obtain

$$
\mathbf{E} \bumpeq V(t, y(t), i) \leq \mathbf{E} \zeta^{T}(t) \Pi_{i} \zeta(t) \leq-\beta_{i} \mathbf{E}|y(t ; \xi)|^{2} \leq-\beta \mathbf{E}|y(t ; \xi)|^{2}
$$

Applying the Dynkin formula and from (3.18), it follows that

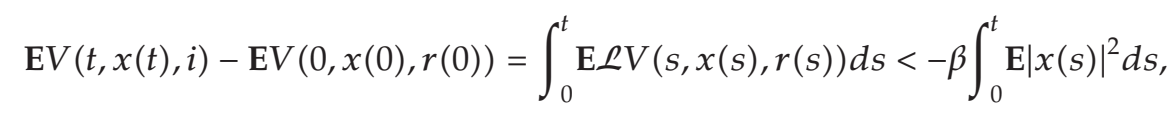

and so

$$
\int_{0}^{t} \mathbf{E}|x(s)|^{2} d s<-\frac{1}{\beta} \mathbf{E} V(t, x(t), i)+\frac{1}{\beta} \mathbf{E} V(0, x(0), r(0))<\frac{1}{\beta} \mathbf{E} V(0, x(0), r(0)),
$$

which implies that the equilibrium point of (2.3) (or (2.2) equivalently) is stochastically asymptotic stability in the mean square. This completes the proof

Remark 3.2. Theorem 3.1 provides a sufficient condition for the generalized neural network (2.3) to ascertain the stochastic asymptotically stability in the mean square of the equilibrium point. The condition is easy to be verified and can be applied in practice as it can be checked by using recently developed algorithms in solving LMIs.

Remark 3.3. The generalized neural network (2.3) is quite general since it considers the effects of many factors including noise perturbations, Markovian jump parameters, and continuously distributed delays. Furthermore, the constants $u_{i}^{-}, v_{i}^{-}, w_{i}^{-}, u_{i}^{+}, v_{i}^{+}, w_{i}^{+}$in Assumption 2.1 are allowed to be positive, negative, or zero. To the best of our knowledge, 
the generalized neural network (2.3) has never been considered in the previous literature. Hence, the LMI criteria existed in all the previous literature fail in our results.

Remark 3.4. If we take $A_{i}=0(i \in S)$, then system (2.3) can be written as

$$
\begin{aligned}
d x(t)= & {\left[-D(r(t)) x(t)+B(r(t)) g(x(t-\tau))+C(r(t)) \int_{-\infty}^{t} R(t-s) h(x(s)) d s+V\right] d t } \\
& +\sigma(x(t), x(t-\tau), t, r(t)) d w(t) .
\end{aligned}
$$

If we take $B_{i}=0(i \in S)$, then system (2.3) can be written as

$$
\begin{aligned}
d x(t)= & {\left[-D(r(t)) x(t)+A(r(t)) f(x(t))+C(r(t)) \int_{-\infty}^{t} R(t-s) h(x(s)) d s+V\right] d t } \\
& +\sigma(x(t), x(t-\tau), t, r(t)) d w(t) .
\end{aligned}
$$

If we do not consider noise perturbations, then system (2.3) can be written as

$$
\begin{aligned}
d x(t)=[ & -D(r(t)) x(t)+A(r(t)) f(x(t)) \\
& \left.+B(r(t)) g(x(t-\tau))+C(r(t)) \int_{-\infty}^{t} R(t-s) h(x(s)) d s+V\right] d t .
\end{aligned}
$$

To the best of our knowledge, even systems (3.21)-(3.23) still have not been investigated in the previous literature.

Remark 3.5. We now illustrate that the neural network (2.3) generalizes some neural networks considered in the earlier literature. For example, if we take

$$
R_{i}(s)=\left\{\begin{array}{ll}
0 & s \geq \tau^{\prime}, \\
1 & s<\tau^{\prime},
\end{array} \tau^{\prime}>0, i=1,2, \ldots, n\right.
$$

then system (2.3) can be written as

$$
\begin{aligned}
d x(t)= & {\left[-D(r(t)) x(t)+A(r(t)) f(x(t))+B(r(t)) g(x(t-\tau))+C(r(t)) \int_{t-\tau^{\prime}}^{t} h(x(s)) d s+V\right] d t } \\
& +\sigma(x(t), x(t-\tau), t, r(t)) d w(t) .
\end{aligned}
$$


System (3.25) was discussed by Liu et al. [26] and Wang et al. [27], although the delays are time-varyings in [27]. Nevertheless, we point out that system (3.25) can be generalized to the neural networks with time-varying delays without any difficulty. If we take $C_{i}=0(i \in S)$ and do not consider noise perturbations, then system (2.3) can be written as

$$
d x(t)=[-D(r(t)) x(t)+A(r(t)) f(x(t))+B(r(t)) g(x(t-\tau))+V] d t
$$

The stability analysis for system (3.26) was investigated by Wang et al. [20]. In [15], Lou and Cui also consider system (3.26) with $A_{i}=0(i \in S)$.

The next five corollaries follow directly from Theorem 3.1, and so we omit their proofs.

Corollary 3.6. Under Assumptions 2.1-2.3, the equilibrium point of (3.21) is stochastic asymptotically stable, if there exist positive scalars $\lambda_{i}$, positive definite matrices $G, M, P_{i}, L_{i}(i \in S)$, and three positive diagonal matrices $Q_{2}, Q_{3}$, and $F=\operatorname{diag}\left(f_{1}, f_{2}, \ldots, f_{n}\right)$ such that the following LMIs hold:

$$
\left.\begin{array}{ccccccc}
\Gamma_{11} & 0 & 0 & 0 & P_{i} B_{i} & P_{i} C_{i} \\
\star & \Gamma_{22} & 0 & 0 & 0 & 0 \\
\star & \star & \Gamma_{44} & 0 & 0 & 0 \\
\star & \star & \star & -Q_{3}+F & 0 & 0 \\
\star & \star & \star & \star & -L_{i} & 0 \\
\star & \star & \star & \star & \star & -F
\end{array}\right]<0
$$

where the symbol " $\star$ " denotes the symmetric term of the matrix:

$$
\begin{gathered}
\Gamma_{11}=-2 P_{i} D_{i}+G+\lambda_{i} \Sigma_{1 i}+V Q_{2} V+W Q_{3} W+\sum_{j=1}^{N} q_{i j} P_{j}, \\
\Gamma_{22}=-G+\lambda_{i} \Sigma_{2 i}, \quad \Gamma_{44}=-Q_{2}+L_{i}+\tau M, \\
V=\operatorname{diag}\left(v_{1}, v_{2}, \ldots, v_{n}\right), \quad W=\operatorname{diag}\left(w_{1}, w_{2}, \ldots, w_{n}\right), \\
v_{i}=\max \left\{\left|v_{i}^{-}\right|,\left|v_{i}^{+}\right|\right\}, \quad w_{i}=\max \left\{\left|w_{i}^{-}\right|,\left|w_{i}^{+}\right|\right\} \quad(i=1,2, \ldots, n) .
\end{gathered}
$$

Corollary 3.7. Under Assumptions 2.1-2.3, the equilibrium point of (3.22) is stochastic asymptotically stable, if there exist positive scalars $\lambda_{i}$, positive definite matrices $G, H, P_{i}, K_{i}(i \in S)$, and three 
positive diagonal matrices $Q_{1}, Q_{3}$, and $F=\operatorname{diag}\left(f_{1}, f_{2}, \ldots, f_{n}\right)$ such that the following LMIs hold:

$$
\left[\begin{array}{cccccc}
\Gamma_{11} & 0 & P_{i} A_{i} & 0 & 0 & P_{i} C_{i} \\
\star & \Gamma_{22} & 0 & 0 & 0 & 0 \\
\star & \star & \Gamma_{33} & 0 & 0 & 0 \\
\star & \star & \star & -Q_{3}+F & 0 & 0 \\
\star & \star & \star & \star & -K_{i} & 0 \\
\star & \star & \star & \star & \star & -F
\end{array}\right]<0,
$$

where the symbol " $\star$ " denotes the symmetric term of the matrix:

$$
\begin{gathered}
\Gamma_{11}=-2 P_{i} D_{i}+G+\lambda_{i} \Sigma_{1 i}+U Q_{1} U+W Q_{3} W+\sum_{j=1}^{N} q_{i j} P_{j}, \\
\Gamma_{22}=-G+\lambda_{i} \Sigma_{2 i}, \quad \Gamma_{33}=-Q_{1}+K_{i}+\tau H, \\
U=\operatorname{diag}\left(u_{1}, u_{2}, \ldots, u_{n}\right), \quad W=\operatorname{diag}\left(w_{1}, w_{2}, \ldots, w_{n}\right), \\
u_{i}=\max \left\{\left|u_{i}^{-}\right|,\left|u_{i}^{+}\right|\right\}, \quad w_{i}=\max \left\{\left|w_{i}^{-}\right|,\left|w_{i}^{+}\right|\right\} \quad(i=1,2, \ldots, n) .
\end{gathered}
$$

Corollary 3.8. Under Assumptions 2.1-2.3, the equilibrium point of (3.23) is stochastic asymptotically stable, if there exist positive scalars $\lambda_{i}$, positive definite matrices $G, H, M, P_{i}, K_{i}, L_{i}(i \in S)$, and three positive diagonal matrices $Q_{1}, Q_{2}$, and $F=\operatorname{diag}\left(f_{1}, f_{2}, \ldots, f_{n}\right)$ such that the following LMIs hold:

$$
\left[\begin{array}{cccccccc}
\Gamma_{11} & 0 & P_{i} A_{i} & 0 & 0 & 0 & P_{i} B_{i} & P_{i} C_{i} \\
\star & -G & 0 & 0 & 0 & 0 & 0 & 0 \\
\star & \star & \Gamma_{33} & 0 & 0 & 0 & 0 & 0 \\
\star & \star & \star & \Gamma_{44} & 0 & 0 & 0 & 0 \\
\star & \star & \star & \star & -Q_{3}+F & 0 & 0 & 0 \\
\star & \star & \star & \star & \star & -K_{i} & 0 & 0 \\
\star & \star & \star & \star & \star & \star & -L_{i} & 0 \\
\star & \star & \star & \star & \star & \star & \star & -F
\end{array}\right]<0,
$$


where the symbol " $\star$ " denotes the symmetric term of the matrix:

$$
\begin{gathered}
\Gamma_{11}=-2 P_{i} D_{i}+G+U Q_{1} U+V Q_{2} V+W Q_{3} W+\sum_{j=1}^{N} q_{i j} P_{j}, \\
\Gamma_{33}=-Q_{1}+K_{i}+\tau H, \quad \Gamma_{44}=-Q_{2}+L_{i}+\tau M, \\
U=\operatorname{diag}\left(u_{1}, u_{2}, \ldots, u_{n}\right), \quad V=\operatorname{diag}\left(v_{1}, v_{2}, \ldots, v_{n}\right), \quad W=\operatorname{diag}\left(w_{1}, w_{2}, \ldots, w_{n}\right), \\
u_{i}=\max \left\{\left|u_{i}^{-}\right|,\left|u_{i}^{+}\right|\right\}, \quad v_{i}=\max \left\{\left|v_{i}^{-}\right|,\left|v_{i}^{+}\right|\right\}, \quad w_{i}=\max \left\{\left|w_{i}^{-}\right|,\left|w_{i}^{+}\right|\right\} \quad(i=1,2, \ldots, n) .
\end{gathered}
$$

Corollary 3.9. Under Assumptions 2.1-2.3, the equilibrium point of (3.25) is stochastic asymptotically stable, if there exist positive scalars $\lambda_{i}$, positive definite matrices $G, H, M, P_{i}, K_{i}, L_{i}(i \in S)$, and four positive diagonal matrices $Q_{1}, Q_{2}, Q_{3}$, and $F=\operatorname{diag}\left(f_{1}, f_{2}, \ldots, f_{n}\right)$ such that the following LMIs hold:

$$
\left[\begin{array}{cccccccc}
\Gamma_{11} & 0 & P_{i} A_{i} & 0 & 0 & 0 & P_{i} B_{i} & P_{i} C_{i} \\
\star & \Gamma_{22} & 0 & 0 & 0 & 0 & 0 & 0 \\
\star & \star & \Gamma_{33} & 0 & 0 & 0 & 0 & 0 \\
\star & \star & \star & \Gamma_{44} & 0 & 0 & 0 & 0 \\
\star & \star & \star & \star & -Q_{3}+F & 0 & 0 & 0 \\
\star & \star & \star & \star & \star & -K_{i} & 0 & 0 \\
\star & \star & \star & \star & \star & \star & -L_{i} & 0 \\
\star & \star & \star & \star & \star & \star & \star & -F
\end{array}\right]<0,
$$

$$
\begin{gathered}
P_{i} \leq \lambda_{i} I, \\
H \geq \sum_{j=1}^{N} q_{i j} K_{j}, \\
M \geq \sum_{j=1}^{N} q_{i j} L_{j},
\end{gathered}
$$

where the symbol " $\star$ " denotes the symmetric term of the matrix:

$$
\begin{gathered}
\Gamma_{11}=-2 P_{i} D_{i}+G+\lambda_{i} \Sigma_{1 i}+U Q_{1} U+V Q_{2} V+W Q_{3} W+\sum_{j=1}^{N} q_{i j} P_{j}, \\
\Gamma_{22}=-G+\lambda_{i} \Sigma_{2 i}, \quad \Gamma_{33}=-Q_{1}+K_{i}+\tau H, \quad \Gamma_{44}=-Q_{2}+L_{i}+\tau M, \\
U=\operatorname{diag}\left(u_{1}, u_{2}, \ldots, u_{n}\right), \quad V=\operatorname{diag}\left(v_{1}, v_{2}, \ldots, v_{n}\right), \quad W=\operatorname{diag}\left(w_{1}, w_{2}, \ldots, w_{n}\right), \\
u_{i}=\max \left\{\left|u_{i}^{-}\right|,\left|u_{i}^{+}\right|\right\}, \quad v_{i}=\max \left\{\left|v_{i}^{-}\right|,\left|v_{i}^{+}\right|\right\}, \quad w_{i}=\max \left\{\left|w_{i}^{-}\right|,\left|w_{i}^{+}\right|\right\} \quad(i=1,2, \ldots, n) .
\end{gathered}
$$


Corollary 3.10. Under Assumption 2.1, the equilibrium point of (3.26) is stochastic asymptotically stable, if there exist positive scalars $\lambda_{i}$, positive definite matrices $G, H, M, P_{i}, K_{i}, L_{i}(i \in S)$, and three positive diagonal matrices $Q_{1}, Q_{2}$, and $F=\operatorname{diag}\left(f_{1}, f_{2}, \ldots, f_{n}\right)$ such that the following LMIs hold:

$$
\begin{aligned}
& {\left[\begin{array}{cccccc}
\Gamma_{11} & 0 & P_{i} A_{i} & 0 & 0 & P_{i} B_{i} \\
\star & -G & 0 & 0 & 0 & 0 \\
\star & \star & \Gamma_{33} & 0 & 0 & 0 \\
\star & \star & \star & \Gamma_{44} & 0 & 0 \\
\star & \star & \star & \star & -K_{i} & 0 \\
\star & \star & \star & \star & \star & -L_{i}
\end{array}\right]<0,} \\
& H \geq \sum_{j=1}^{N} q_{i j} K_{j}, \\
& M \geq \sum_{j=1}^{N} q_{i j} L_{j},
\end{aligned}
$$

where the symbol " $\star$ " denotes the symmetric term of the matrix:

$$
\begin{gathered}
\Gamma_{11}=-2 P_{i} D_{i}+G+U Q_{1} U+V Q_{2} V+\sum_{j=1}^{N} q_{i j} P_{j}, \\
\Gamma_{33}=-Q_{1}+K_{i}+\tau H, \quad \Gamma_{44}=-Q_{2}+L_{i}+\tau M, \\
U=\operatorname{diag}\left(u_{1}, u_{2}, \ldots, u_{n}\right), \quad V=\operatorname{diag}\left(v_{1}, v_{2}, \ldots, v_{n}\right), \\
u_{i}=\max \left\{\left|u_{i}^{-}\right|,\left|u_{i}^{+}\right|\right\}, \quad v_{i}=\max \left\{\left|v_{i}^{-}\right|,\left|v_{i}^{+}\right|\right\} \quad(i=1,2, \ldots, n) .
\end{gathered}
$$

Remark 3.11. As discussed in Remark 3.4, Corollaries 3.6-3.8 are "new" since they have never been considered in the previous literature. Corollary 3.9 was discussed by Liu et al. [26] and Wang et al. [27], although the delays are time-varyings in [27]. Nevertheless, we point out that our results can be generalized to the neural networks with time-varying delays without any difficulty. Corollary 3.10 has been discussed by Wang et al. [20] and Lou and Cui [15], but our conditions are weaker than those in $[15,20]$, for the constants $u_{i}^{-}, v_{i}^{-}, u_{i}^{+}, v_{i}^{+}$in Corollary 3.10 are allowed to be positive, negative, or zero.

\section{Illustrative Example}

In this section, a numerical example is given to illustrate the effectiveness of the obtained results. 
Example 4.1. Consider a two-dimensional stochastic neural network with both Markov jump parameters and continuously distributed delays:

$$
\begin{aligned}
d x(t)=[ & -D(r(t)) x(t)+A(r(t)) f(x(t))+B(r(t)) g(x(t-\tau)) \\
& \left.+C(r(t)) \int_{-\infty}^{t} e^{-(t-s)} h(x(s)) d s+V\right] d t+\sigma(x(t), x(t-\tau), t, r(t)) d w(t),
\end{aligned}
$$

where $x(t)=\left(x_{1}(t), x_{2}(t)\right)^{T}, V=(0,0)^{T}, w(t)$ is a two dimensional Brownian motion, and $r(t)$ is a right-continuous Markov chain taking values in $S=\{1,2\}$ with generator

$$
Q=\left[\begin{array}{cc}
-8 & 8 \\
5 & -5
\end{array}\right]
$$

Let

$$
f_{i}\left(x_{i}\right)=0.01 \tan h x_{i}=\frac{0.01\left(e^{x_{i}}-e^{-x_{i}}\right)}{\left(e^{x_{i}}+e^{-x_{i}}\right)}, \quad g_{i}\left(x_{i}\right)=h_{i}\left(x_{i}\right)=0.005\left(\left|x_{i}+1\right|-\left|x_{i}-1\right|\right) \quad(i=1,2),
$$

then system (4.1) satisfies Assumption 2.1 with $U^{-}=V^{-}=W^{-}=-0.01 I, U^{+}=V^{+}=W^{+}=$ 0.01I. Take

$$
\sigma(x(t), x(t-\tau), t, i)=\left(\begin{array}{ll}
0.2 x_{i}(t) & 0.4 x_{i}(t-\tau) \\
0.4 x_{i}(t) & 0.2 x_{i}(t-\tau)
\end{array}\right) \quad(i=1,2)
$$

then system (4.1) satisfies Assumptions 2.2 and 2.3 with $\Sigma_{1 i}=\Sigma_{2 i}=0.2 I(i=1,2)$.

Other parameters of the network (4.1) are given as follows:

$$
\begin{aligned}
& D_{1}=\operatorname{diag}(0.3,0.1), \quad A_{1}=\left[\begin{array}{cc}
0.2 & -0.2 \\
0.4 & 0.1
\end{array}\right], \quad B_{1}=\left[\begin{array}{cc}
0.1 & -0.2 \\
0.3 & 0.2
\end{array}\right], \quad C_{1}=\left[\begin{array}{cc}
0.1 & 0.2 \\
-0.3 & 0.3
\end{array}\right], \\
& D_{2}=\operatorname{diag}(0.4,0.3), \quad A_{2}=\left[\begin{array}{cc}
0.3 & 0.2 \\
-0.1 & 0.2
\end{array}\right], \quad B_{2}=\left[\begin{array}{cc}
0.2 & 0.1 \\
-0.2 & 0.1
\end{array}\right], \quad C_{2}=\left[\begin{array}{cc}
0.2 & 0.2 \\
-0.3 & 0.1
\end{array}\right] \text {. }
\end{aligned}
$$


Here we let $\tau=2$. By using the Matlab LMI toolbox, we can obtain the following feasible solution for the LMIs (3.1)-(3.4):

$$
\begin{gathered}
G=\left[\begin{array}{cc}
18.71 & 1.41 \\
1.41 & 4.68
\end{array}\right], \quad H=\left[\begin{array}{ll}
39.69 & -6.32 \\
-6.32 & 44.13
\end{array}\right], \\
M=\left[\begin{array}{cc}
29.85 & -1.07 \\
-1.07 & 33.32
\end{array}\right], \quad E=\operatorname{diag}(55.09,55.09), \\
Q_{1}=\left[\begin{array}{cc}
224.65 & 0 \\
0 & 224.65
\end{array}\right], \quad Q_{2}=\left[\begin{array}{cc}
163.84 & 0 \\
0 & 163.84
\end{array}\right], \\
Q_{3}=\left[\begin{array}{cc}
72.69 & 0 \\
0 & 72.69
\end{array}\right], \quad F=\operatorname{diag}(31.88,31.88), \\
P_{1}=\left[\begin{array}{cc}
57.77 & 1.91 \\
1.91 & 39.25
\end{array}\right], \quad P_{2}=\left[\begin{array}{cc}
56.59 & 1.66 \\
1.66 & 37.41
\end{array}\right], \quad K_{1}=\left[\begin{array}{cc}
46.70 & -4.19 \\
-4.19 & 49.63
\end{array}\right], \quad \lambda_{1}=83.25, \\
K_{2}=\left[\begin{array}{cc}
46.40 & -4.17 \\
-4.17 & 49.33
\end{array}\right], \quad L_{1}=\left[\begin{array}{cc}
64.95 & 3.06 \\
3.06 & 55.17
\end{array}\right], \quad L_{2}=\left[\begin{array}{cc}
64.41 & 2.99 \\
2.99 & 54.64
\end{array}\right], \quad \lambda_{2}=79.06 .
\end{gathered}
$$

Therefore, it follows from Theorem 3.1 that the network (4.1) is stochastic asymptotically stable.

By using the Euler-Maruyama numerical scheme, simulation results are as follows: $T=50$ and step size $\delta t=0.02$. Figure 1 is the state response of model 1 (i.e., the network (4.1) when $r(t)=1$ ) with the initial condition $[0.5,-0.7]^{T}$, for $-2 \leq t \leq 0$, and Figure 2 is the state response of model 2 (i.e., the network $(4.1)$ when $r(t)=2$ ) with the initial condition $[-0.6,0.4]^{T}$, for $-2 \leq t \leq 0$.

Remark 4.2. As discussed in Remarks 3.2-3.11, the LMI criteria existed in all the previous literature (e.g., Liu et al. [26], Wang et al. [20, 27], Lou and Cui [15], etc.) fail in Example 4.1 since many factors including noise perturbations, Markovian jump parameters, and continuously distributed delays are considered in Example 4.1.

\section{Concluding Remarks}

In this paper we have investigated the stochastic stability analysis problem for a class of neural networks with both Markovian jump parameters and continuously distributed delays. It is worth mentioning that our obtained stability condition is delay-dependent, which is less conservative than delay-independent criteria when the delay is small. Furthermore, the obtained stability criteria in this paper are expressed in terms of LMIs, which can be solved easily by recently developed algorithms. A numerical example is given to show the less 


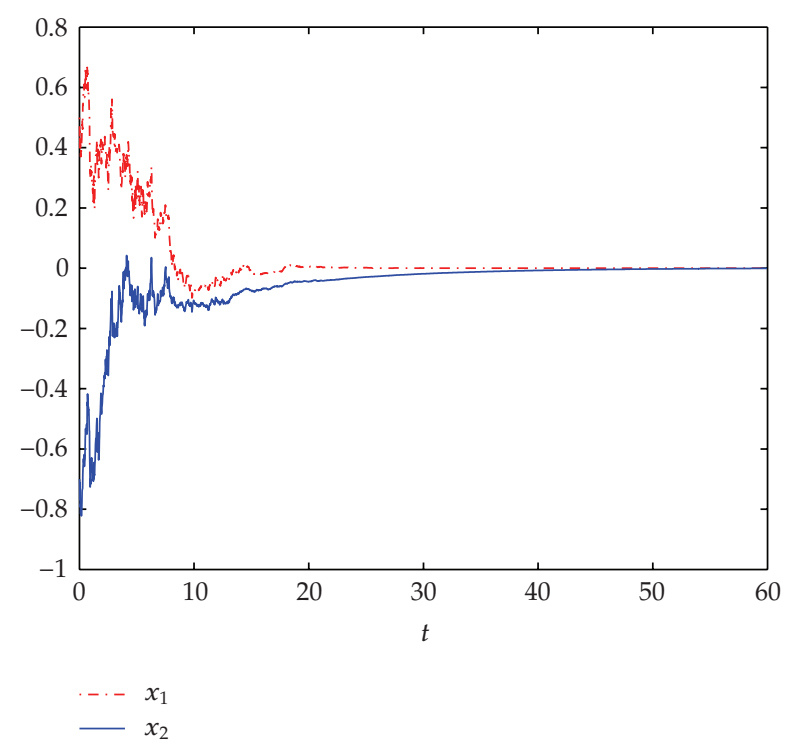

Figure 1: The state response of the model 1 in Example 4.1.

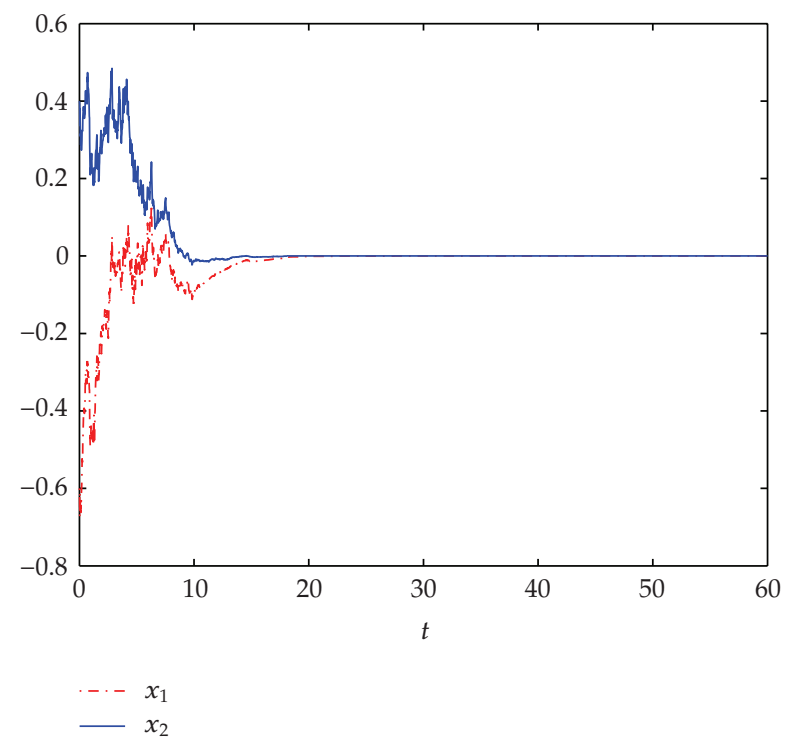

Figure 2: The state response of the model 2 in Example 4.1.

conservatism and effectiveness of our results. The results obtained in this paper improve and generalize those given in the previous literature. On the other hand, it should be noted that the explicit rate of convergence for the considered system is not given in this paper since it is difficult to deal with continuously distributed delays. Therefore, investigating the explicit rate of convergence for the considered system remains an open issue. Finally, we point out that it is possible to generalize our results to a class of neural networks with uncertainties. Research on this topic is in progress. 


\section{Acknowledgments}

The authors would like to thank the editor and five anonymous referees for their helpful comments and valuable suggestions regarding this paper. This work was jointly supported by the National Natural Science Foundation of China $(10801056,60874088)$, the Natural Science Foundation of Guangdong Province (06300957), K. C. Wong Magna Fund in Ningbo University, and the Specialized Research Fund for the Doctoral Program of Higher Education (20070286003).

\section{References}

[1] A. Cichocki and R. Unbehauen, Neural Networks for Optimalition and Signal Processing, John Wiley \& Sons, New York, NY, USA, 1993.

[2] L. O. Chua and L. Yang, "Cellular neural networks: applications," IEEE Transactions on Circuits and Systems, vol. 35, no. 10, pp. 1273-1290, 1988.

[3] G. Joya, M. A. Atencia, and F. Sandoval, "Hopfield neural networks for optimization: study of the different dynamics," Neurocomputing, vol. 43, pp. 219-237, 2002.

[4] W.-J. Li and T. Lee, "Hopfield neural networks for affine invariant matching," IEEE Transactions on Neural Networks, vol. 12, no. 6, pp. 1400-1410, 2001

[5] S. S. Young, P. D. Scott, and N. M. Nasrabadi, "Object recognition using multilayer Hopfield neural network," IEEE Transactions on Image Processing, vol. 6, no. 3, pp. 357-372, 1997.

[6] S. Arik, "Stability analysis of delayed neural networks," IEEE Transactions on Circuits and Systems I, vol. 47, no. 7, pp. 1089-1092, 2000.

[7] S. Blythe, X. Mao, and X. Liao, "Stability of stochastic delay neural networks," Journal of the Franklin Institute, vol. 338, no. 4, pp. 481-495, 2001.

[8] J. Cao, A. Chen, and X. Huang, "Almost periodic attractor of delayed neural networks with variable coeffcients," Physics Letters A, vol. 340, no. 1-4, pp. 104-120, 2005.

[9] J. Cao and J. Wang, "Global exponential stability and periodicity of recurrent neural networks with time delays," IEEE Transactions on Circuits and Systems I, vol. 52, no. 5, pp. 920-931, 2005.

[10] W.-H. Chen and X. Lu, "Mean square exponential stability of uncertain stochastic delayed neural networks," Physics Letters A, vol. 372, no. 7, pp. 1061-1069, 2008.

[11] W.-H. Chen and W. X. Zheng, "Global asymptotic stability of a class of neural networks with distributed delays," IEEE Transactions on Circuits and Systems I, vol. 53, no. 3, pp. 644-652, 2006.

[12] H. Huang, D. W. C. Ho, and J. Lam, "Stochastic stability analysis of fuzzy Hopeld neural networks with time-varying delays," IEEE Transactions on Circuits and Systems II, vol. 52, no. 5, pp. 251-255, 2005.

[13] M. P. Joy, "Results concerning the absolute stability of delayed neural networks," Neural Networks, vol. 13, no. 6, pp. 613-616, 2000.

[14] Y. Liu, Z. Wang, and X. Liu, “On global exponential stability of generalized stochastic neural networks with mixed time-delays," Neurocomputing, vol. 70, no. 1-3, pp. 314-326, 2006.

[15] X. Lou and B. Cui, "Delay-dependent stochastic stability of delayed Hopfield neural networks with Markovian jump parameters," Journal of Mathematical Analysis and Applications, vol. 328, no. 1, pp. 316-326, 2007.

[16] X. Mao, Stochastic Differential Equations and Their Applications, Horwood Publishing Series in Mathematics \& Applications, Horwood Publishing, Chichester, UK, 1997.

[17] Y. S. Moon, P. Park, W. H. Kwon, and Y. S. Lee, "Delay-dependent robust stabilization of uncertain state-delayed systems," International Journal of Control, vol. 74, no. 14, pp. 1447-1455, 2001.

[18] J. H. Park, "On global stability criterion of neural networks with continuously distributed delays," Chaos, Solitons E Fractals, vol. 37, no. 2, pp. 444-449, 2008.

[19] R. Rakkiyappan and P. Balasubramaniam, "Delay-dependent asymptotic stability for stochastic delayed recurrent neural networks with time varying delays," Applied Mathematics and Computation, vol. 198 , no. 2, pp. 526-533, 2008.

[20] Z. Wang, Y. Liu, L. Yu, and X. Liu, "Exponential stability of delayed recurrent neural networks with Markovian jumping parameters," Physics Letters A, vol. 356, no. 4-5, pp. 346-352, 2006.

[21] L. Wan and J. Sun, "Mean square exponential stability of stochastic delayed Hopfield neural networks," Physics Letters A, vol. 343, no. 4, pp. 306-318, 2005. 
[22] Q. Zhou and L. Wan, “Exponential stability of stochastic delayed Hopfield neural networks," Applied Mathematics and Computation, vol. 199, no. 1, pp. 84-89, 2008.

[23] P. Baldi and A. F. Atiya, "How delays affect neural dynamics and learning," IEEE Transactions on Neural Networks, vol. 5, no. 4, pp. 612-621, 1994.

[24] S. Boyd, L. El Ghaoui, E. Feron, and V. Balakrishnan, Linear Matrix Inequalities in System and Control Theory, vol. 15 of SIAM Studies in Applied Mathematics, SIAM, Philadelphia, Pa, USA, 1994.

[25] H. Yang and T. Chu, "LMI conditions for stability of neural networks with distributed delays," Chaos, Solitons \& Fractals, vol. 34, no. 2, pp. 557-563, 2007.

[26] Y. Liu, Z. Wang, and X. Liu, “On delay-dependent robust exponential stability of stochastic neural networks with mixed time delays and Markovian switching," Nonlinear Dynamics, vol. 54, no. 3, pp. 199-212, 2008.

[27] G. Wang, J. Cao, and J. Liang, "Exponential stability in the mean square for stochastic neural networks with mixed time-delays and Markovian jumping parameters," Nonlinear Dynamics, vol. 57, no. 1-2, pp. 209-218, 2009. 


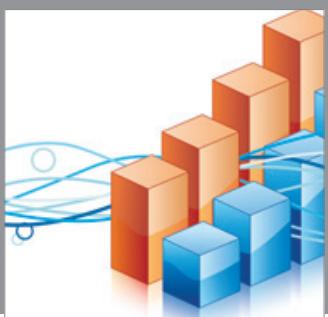

Advances in

Operations Research

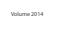

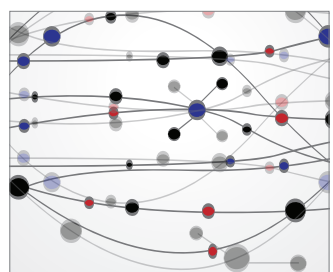

\section{The Scientific} World Journal
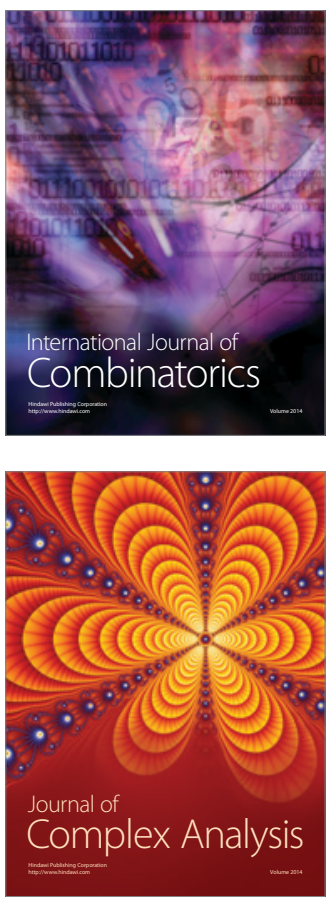

International Journal of

Mathematics and

Mathematical

Sciences
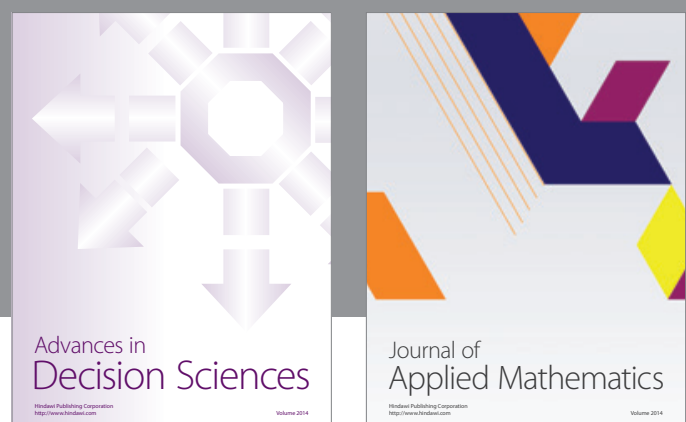

Journal of

Applied Mathematics
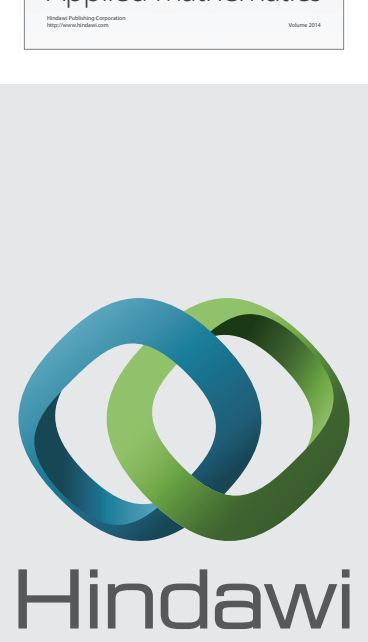

Submit your manuscripts at http://www.hindawi.com
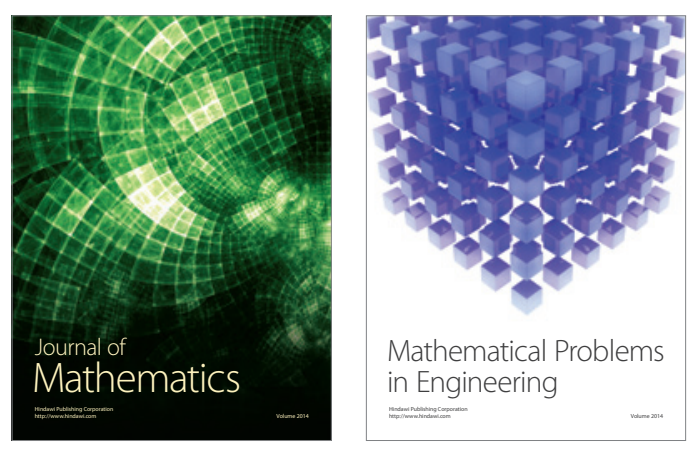

Mathematical Problems in Engineering
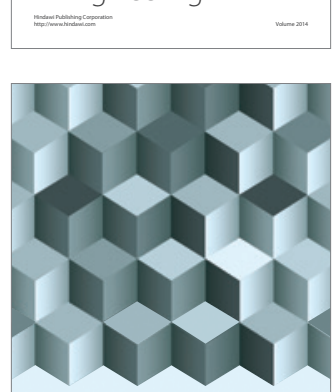

Journal of

Function Spaces
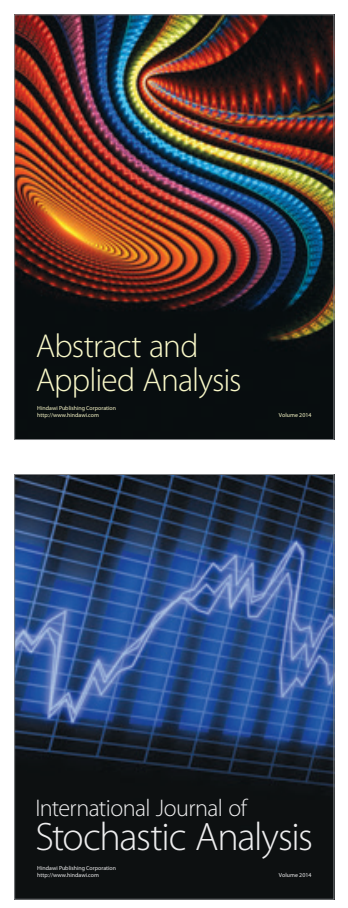

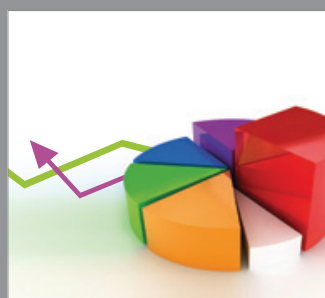

ournal of

Probability and Statistics

Promensencen
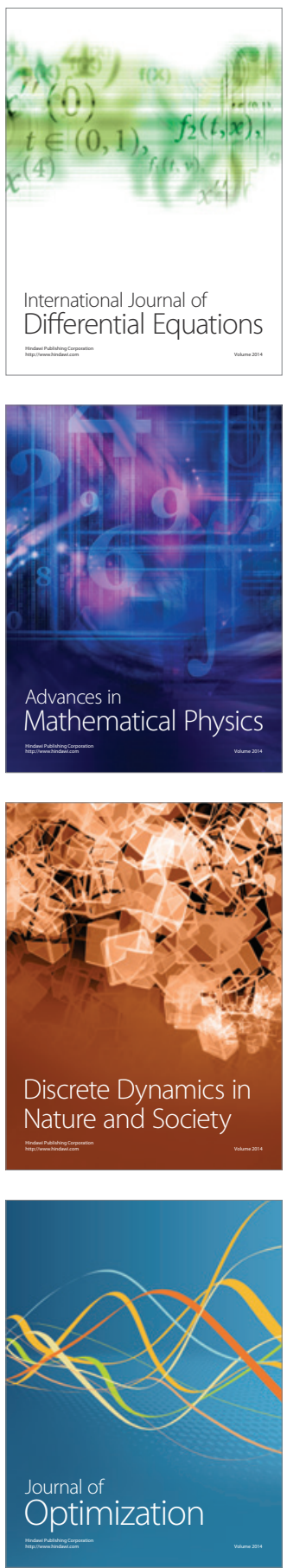